\title{
Application of Lippmann interference photography to data storage
}

\section{Kevin Contreras}

\section{Gilles Pauliat}

gilles.pauliat@institutoptique.fr

\section{Carole Arnaud}

\section{Gérald Roosen}

\begin{abstract}
Laboratoire Charles Fabry de l'Institut d'Optique, CNRS, Univ Paris-Sud, Campus Polytechnique, RD 128, 91127 Palaiseau cedex, France

Laboratoire Charles Fabry de l'Institut d'Optique, CNRS, Univ Paris-Sud, Campus Polytechnique, RD 128, 91127 Palaiseau cedex, France

Laboratoire Charles Fabry de l'Institut d'Optique, CNRS, Univ Paris-Sud, Campus Polytechnique, RD 128, 91127 Palaiseau cedex, France

Laboratoire Charles Fabry de l'Institut d'Optique, CNRS, Univ Paris-Sud, Campus Polytechnique, RD 128, 91127 Palaiseau cedex, France
\end{abstract}

Lippmann's interference photography is an elegant process to record coloured images in the volume of a sensitive material. We propose to use this technique for wavelength multiplexed data storage in a page-oriented approach. Using computer simulations, we demonstrate that the capacities reached with this technique are similar to those reached by volume holographic data storage. [D0I: 10.2971/je0s.2008.08020]

Keywords: optical data storage: holographic and volume memories, holography: volume gratings.

\section{INTRODUCTION}

Holographic data storage is considered as one of the most serious candidates for the next generation of high capacity optical disks [1]. Holographic storage has indeed already proved its ability to reach very large data capacities, and roadmaps to achieve capacities beyond one TeraByte within a few years are already well commented [2]. In addition to this advantage in term of capacity, holography presents an inherent parallelism of data, which follows the fact that, in most architectures, data are aggregated in images for recording and readout. This parallelism is at the origin of the expected high recording and readout data rates [3].

Besides all this interest aroused by the application of holography to optical storage, we believe that simpler optical arrangements based on Lippmann's photography have not been studied with enough attention. The goal of this paper is thus to describe an optical data storage arrangement based on the Lippmann's architecture. Using numerical simulations, we investigate the storage capacity of these systems and show that they can be as large as those achievable by holography.

\section{FROM LIPPMANN'S COLOUR INTERFERENCE PHOTOGRAPHY...}

Lippmann's colour photography, also known as interference colour photography, is a more than one century old process. Gabriel Lippmann published his first work in 1891 [4] and got the Nobel price for this discovery in 1908. Lippmann's photography is a very elegant technique based on the recording of interferences [5]-[7]. The coloured objects or landscapes to be recorded are imaged through the thick photographic emulsion onto a mirror set in contact with this emulsion (see Fig- ure 1). For each pixel of the image, the incident beam thus interferes with its reflection and records a small Bragg grating inside the thickness of the sensitive layer. The mirror is then removed and the recording plate is chemically processed. Illuminating the processed layer at normal incidence with white light reproduces the image: for each pixel of the image, the Bragg gratings retro-reflect the exact wavelengths previously used for data recording. The observer experiences the true colours by looking at the plate at normal incidence.

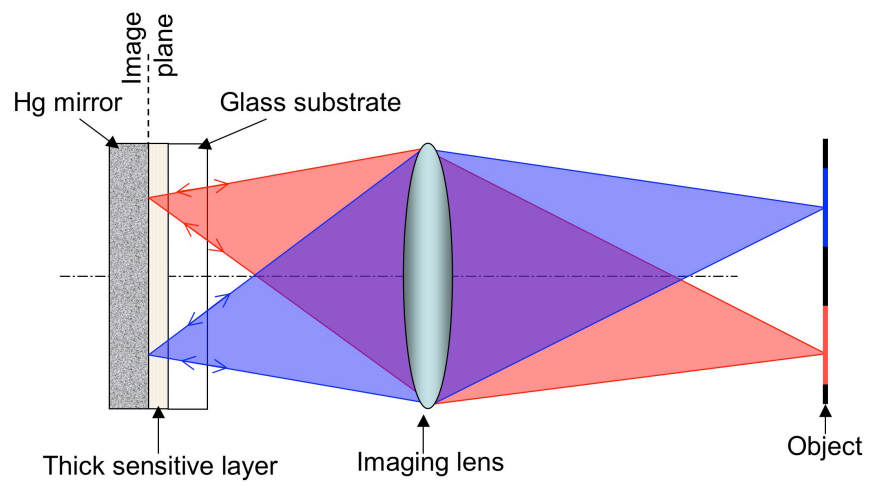

FIG. 1 Principle of Lippmann's photography.

In spite of its elegance, shooting Lippmann's photographs requires very skilled experimentalists, which partly explains the nearly complete dying out of this process at the beginning of the twentieth century at the benefit of other colour photographic processes, such as the "Autochromes". Nevertheless a lot of developments felt out of this technique, which is thus at the origin of several other scientific breakdowns. Yuri N. Denisyuk's reflection holography is for instance a tremen- 
dous invention based on Lippmann's photography [8, 9]. Indeed, although Lippmann records interferences in the volume of the material, the phase of the original object is not recorded in a Lippmann plate. The recorded images only bear 2-D information, there is no 3D information. In this process, the mirror indeed acts as a phase locker, that is distinct from the object, and the phase of the beam, which is reproduced during readout, is just the incident phase plus a constant depending on the mirror properties ( $a \pi$ phase shift in case of the mercury mirror used by Lippmann). In case of Denisyuk's holography, the phase of the diffracted beam bears the information on the position of each point of the object: the relief of the original object is reconstructed during readout.

It is worth noting that holography led to a large amount of scientific works in the area of data storage because of its ability to record data in the volume of the material, although in most set-ups, the recording of the phase of the object is not the key point $[3,10,11]$. Indeed the data to be recorded are usually imprinted on the incident beam with an intensity spatial light modulator, SLM, and are detected during readout by an array of intensity detectors such as a CCD or a CMOS camera. If the phase information is nevertheless sometimes shrewdly used to simplify the architectures, such as in the phase conjugate architectures [3] or in phase multiplexing [12], it is not essential to the storage process.

Considering this fact, it is interesting to reexamine Lippmann's photographic process in view of achieving a high capacity volume data storage system.

\section{3 ... TO HIGH CAPACITY DATA STORAGE}

Original Lippmann's photographs are snapped with natural light whose coherence length is at a maximum equal to a few micrometers. This is the reason why Lippmann's plates are usually less than $10 \mu \mathrm{m}$ thick and for which a mercury mirror is used. This mercury mirror is a very convenient way to insure an intimate contact with the emulsion and to be sure that the zero order fringe is recorded inside the emulsion.

Today, the availability of light sources with longer coherence lengths allows us to revisit this recording process and to use much thicker recording layers.

The idea of applying this recording process to data storage is in fact not new and was applied in the mid 60 s by Fleisher et al. to record binary data [13]. In this demonstration, a photographic plate with its sensitive emulsion in contact with a mercury layer was used just as in the original arrangement proposed by Gabriel Lippmann. Data were first imprinted on transmission masks: each bit in the " 1 " state corresponding to a transparent pixel of the mask, the " 0 " corresponding to opaque pixels. Several data masks were successively recorded in the sensitive layer by illuminating the Lippmann structure through the data masks with successive wavelengths selected from an arc source filtered by a set of interference filters. Bragg microgratings are thus recorded at different wavelengths in the same location of the photographic emulsion, thus achieving wavelength Bragg multiplexed micro-grating data recording. Although this first demonstration was successful, the storage capacity was greatly limited by the optical sources and by the photographic plates available at that time. Indeed the wet processing, inherent to photographic plates, leads to an inhomogeneous swelling through the depth of the emulsion and thus strongly reduces the wavelength Bragg selectivity. This non-uniform swelling limits the material thickness and thus the data capacity.

With the advent of the first laser diodes, a new compact architecture was proposed [14]. Nevertheless, this demonstration was still performed with a photographic plate, with the inherent limitations due to the wet processing.

More recently the development of new holographic materials, such as photopolymers, eased the experimental demonstrations of Lippmann data storage systems [15]-[19]. Although quite interesting, all these previous demonstrations are bitoriented data architectures: during recording, the lights from adjacent bits are prevented from interfering, for instance by confining the light of each pixel in a micro-fibre [15].

In this paper, we investigate a completely different approach: we are interested in page-oriented architectures (Figure 2(a) and $2(b))$.

Bits of data are first imprinted by a spatial light modulator on the beam. This modulator is imaged onto the Lippmann's mirror set beneath the photosensitive layer. This beam, at normal incidence, interferes with its reflection and thus records a complex Bragg grating in the sensitive layer. In this geometry, the material thickness can be larger than the depth of focus of the image so that lights from the various pixels are allowed to interfere. This point is important as this is the key to obtain large diffraction efficiencies, and as we will demonstrate, to get wavelength selective gratings. Several images can be successively recorded at the same location using wavelength multiplexing, i.e. each image is recorded at a specific wavelength.

Each image should be reconstructed with a plane wave readout beam set at the wavelength used for recording this very same image. We expect that the cross-talk between images can be made negligible by correctly selecting the spacing between the successive wavelengths as we will describe in the simulations. We also expect that the images are reconstructed with low distortion. This last point is not trivial. We speak about distortion and not noise, as this distortion is deterministic. It is inherent to the Lippmann's process and results from the differences between the reference beam used during recording (reflected image beam) and the plane wave used for reading out. The origin of this noise can be clearly identified by comparing the basic equations for Lippmann's storage and for Denisyuk's holography. For the selected optical arrangement, using complex notations, the electric field $E_{\text {data }}(x, y, z)$ of the image beam incident onto the mirror, and the readout beam electric field $E_{R e f}(x, y, z)$ can be written as:

$$
\begin{aligned}
& E_{\text {data }}(x, y, z)=A(x, y, z) \exp \left[-i k_{0} z\right] \\
& E_{\text {Ref }}(x, y, z)=R(x, y, z) \exp \left[-i k_{1} z\right]
\end{aligned}
$$



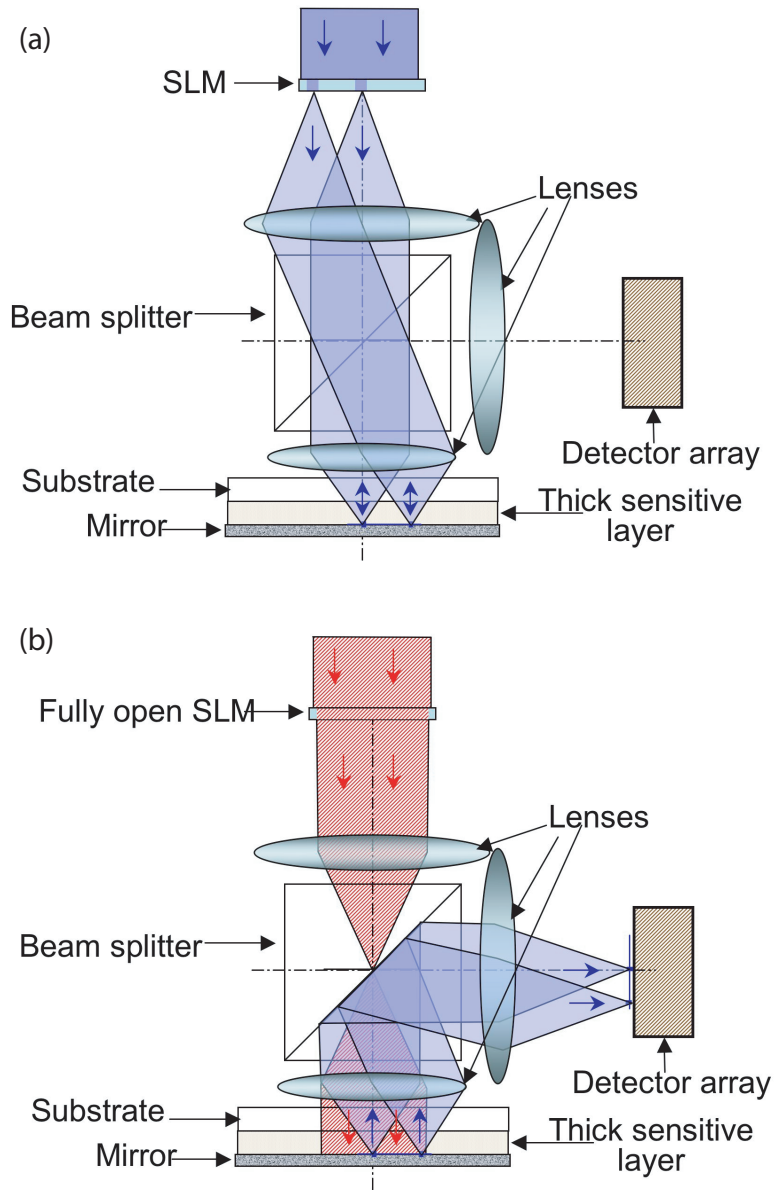

FIG. 2 Example of a data storage system based on Lippmann's architecture; (a) recording operation, for sake of simplicity only two pixels of the SLM are shown open; (b) readout operation, the SLM is either fully opened or removed, for clarity and although being at the same wavelengths, the readout wave is shown in red while the diffracted beam for the two pixels is coloured in blue.

with $k_{0}$ and $k_{1}$ the wave numbers during recording and readout. $z$ is the coordinate along the normal of the mirror; $z=0$ corresponds to the image plane onto the mirror.

In the Lippmann's arrangement, because the image beam is set at normal incidence and because the wavefront is planar at the mirror level, the reflected beam is just its phase conjugate. This is this amplitude $A^{*}$ that we want to reconstruct by diffraction. In Denisyuk's holography, this wave $A^{*}$ originates from the scattering of the plane wave onto the object and directly interferes with this plane wave inside the medium. The interference pattern of these two counterpropagating beams during recording is thus proportional to $A(x, y, z)^{*} B(x, y, z)^{*} \exp \left[2 i k_{0} z\right]$. For Lippmann's approach $B=A$ while in Denisyuk's holography $B=R$. Taking into account the low diffraction efficiencies common in holographic memories, thus applying the first Born's approximation, the amplitude in each layer $z_{0}$ of the medium and observed at $z=z_{0}$ can be expressed by:

$$
E_{\text {diff }}^{z_{0}}\left(x, y, z=z_{0}\right) \propto \delta \varepsilon\left(x, y, z_{0}\right) R\left(x, y, z_{0}\right) \exp \left[-i k_{1} z_{0}\right]
$$

Taking the simplest case in which the permittivity $\delta \varepsilon$ is proportional to the interference pattern, one gets the basic equation describing the diffracted electric field by each layer of the thick medium:

$$
\begin{aligned}
E_{\text {diff }}^{z_{0}}\left(x, y, z=z_{0}\right) \propto A & \left(x, y, z_{0}\right)^{*} B\left(x, y, z_{0}\right)^{*} \\
& \times R\left(x, y, z_{0}\right) \exp \left[i\left(2 k_{0}-k_{1}\right) z_{0}\right]
\end{aligned}
$$

In case of Denisyuk's holography, plane wave $R$ is used for recording, $R=B$. The intensity $R^{*} R$ is just a constant, independent of coordinates $x$ and $y$. This intensity is a proportionality constant in Eq. (3) so that the diffracted amplitude is proportional to $A\left(x, y, z_{0}\right)^{*}$ and the image is perfectly reconstructed. In case of Lippmann's process, the recording wave $B$ being different from the one used for reconstruction, $R$, the proportionality is lost and the retrieved image is distorted.

In order to minimize this distortion, the readout wave should be as similar as possible to the data beam. Typically, $R(x, y, z=0)$ is selected to have the same phase wavefront than $A(x, y, z=0)$. For instance, if the wavefront of the image beam is planar at the mirror level, then the reference beam is a plane wave; because all pixels in the "ON" state have the same intensity, the intensity of the reference beam is uniform. In the following modelling, we assume that this image wavefront is planar at the mirror level. The readout beam is thus also a plane wave at normal incidence. This is important as the Lippmann's process does not record the phase of the incident beam, it cannot reproduce the phase of the diffracted beam. The phase of the readout beam should thus match the phase of the image beam. Nevertheless, other arrangements are possible taking into account the requirement of the phase matching.

\section{MODELLING}

To model our system, we assume low diffraction efficiencies, that is the first Born's approximation, and rely on a plane wave decomposition of the amplitudes of the beams to propagate these amplitudes using Fast Fourier Transform algorithms, FFT [20, 21].

At the initial stage of the modelling, we first determine the image amplitude $A(x, y, z=0)$ and the reference beam amplitude $R(x, y, z=0)$ in plane $z=0$. For doing this, we assume the image beam to be pixellated with square pixels whose sides are denoted $a$. They are arranged in $N \times N$ square matrix with a pitch denoted $s$.

Although, this does not correspond to any optimization, we selected the data by a random draw with equiprobality between 1 and 0 . As in the real system, this original page of data is then filtered: we decompose the amplitude in plane waves and we apply a filter in the Fourier plane. In all the following modelling, this Fourier filter corresponds to an optical system with a $N A=0.6$ numerical aperture.

As explained above the reference beam is a plane wave. In order to minimize the distortion, this plane wave is limited to a square whose side is the same as the pixel matrix side. This amplitude is then filtered by the $N A=0.6$ in order to get $R(x, y, z=0)$.

In a second step, for each coordinate $z_{0}$, we propagate, us- 
ing FFT, amplitude $A(x, y, z=0)$ to get $A\left(x, y, z_{0}\right)$ and the dielectric permittivity $\delta \varepsilon\left(x, y, z_{0}\right)$. To compute this permittivity, we have to assume the response of the material. We made simulations with both a linear response (i.e. proportional to $\left.A\left(x, y, z_{0}\right)^{*} A\left(x, y, z_{0}\right)^{*} \exp \left[2 i k_{0} z_{0}\right]\right)$ or with a response proportional to the modulation ratio of the interference pattern:

$$
\delta \varepsilon\left(x, y, z_{0}\right) \propto \frac{A\left(x, y, z_{0}\right)^{*} A\left(x, y, z_{0}\right)^{*}}{\left\|A\left(x, y, z_{0}\right)\right\|^{2}+\left\|A\left(x, y, z_{0}\right)\right\|^{2}} \exp \left[2 i k_{0} z_{0}\right]
$$

The results obtained with the two responses do not significantly differ from each other, and the conclusions that can be drawn from these simulations are consequently the same. In the following examples, we have taken the response given by Eq. (4). The reference beam amplitude is similarly propagated from coordinate $z=0$ to $z_{0}$.

Using Eq. (3), with $B=A$, the amplitude $E_{\text {diff }}^{z_{0}}\left(x, y, z=z_{0}\right)$ diffracted by the corresponding layer is then calculated. It is then back propagated until the image plane, at $z=0$, to get $E_{\text {diff }}^{z_{0}}(x, y, z=0)$. All the amplitudes $E_{\text {diff }}^{z_{0}}(x, y, z=0)$ are then summed over $z_{0}$. The total amplitude is then filtered by the $N A=0.6$ numerical aperture. The resulting amplitude thus corresponds to the one detected by a detector set on the diffracted beam path and imaged onto the plane $z=0$.

\section{RESULTS OF SIMULATION}

\subsection{Example of retrieved image and distortion}

Unless expressed otherwise, in all simulations presented in this paper, we have selected the following set of parameters: average refractive index $n=1.6$; recording wavelength in vacuum $\lambda=500 \mathrm{~nm}$. The absorption of the material is assumed to be negligible. As already detailed, the optical system is assumed to have a pupil corresponding to a numerical aperture of $N A=0.6$. The pixel side, $a=0.7 \mu \mathrm{m}$, is thus close to the optical resolution of the system. The pitch between centres of pixels is slightly larger than the pixel side: $s=0.76 \mu \mathrm{m}$. It corresponds to a spatial light modulator with a filling factor of 0.85 . We assume that the filling factor of the detector array is the same as the one of the spatial light modulator. The thick recording material is sampled every tenth of micrometer (distance between $z_{0}$ planes) along the $z$ direction. We have checked that a denser sampling does not modify the results within the accuracy of the reported results. In order to get reasonable computing times, we made simulations on a limited volume of the thick recording material. The number of pixels of the SLM is equal to $41^{2}=1681$ pixels. As described below, this limited number of pixels enhances the edge effects: a significant number of pixels are not surrounded by other ones. The observation window along the $x \times y$ coordinates is taken larger than the image size so that, given the 0.6 numerical aperture and the material thickness, the beams are not truncated by the borders of this window. It is equal to $50 \times 50 \mu \mathrm{m}^{2}$. Along these $x \times y$ coordinates, the distances are sampled by $780 \times 780$ samples to perform the FFT, that is $11 \times 11$ samples per SLM pixel.

The simulations reported in Figure 3 are for a material whose thickness is $e=30 \mu \mathrm{m}$. This thickness is much larger than the depth of field of the image $(\approx 2 \mu \mathrm{m})$ so that lights corresponding to adjacent pixels interfere in the volume of the material. Figure 3 corresponds to the original image to be recorded, displayed on the SLM, but before being filtered by the numerical aperture of the optical system projecting this image onto the mirror. The retrieved diffracted intensity image filtered by the optical system is shown in Figure 4.

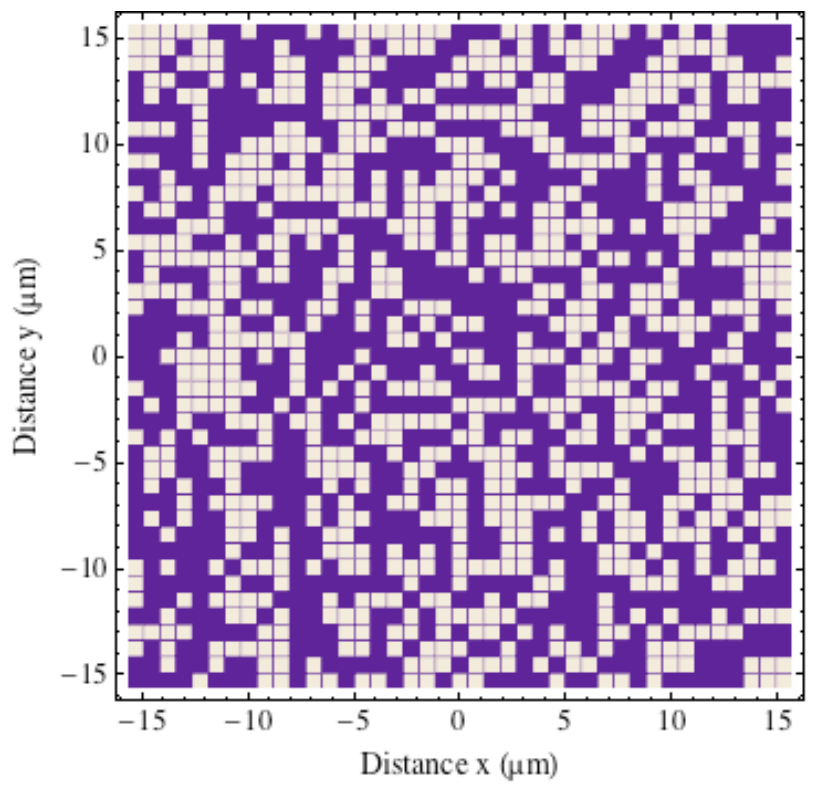

FIG. 3 Density plot of the image before recording.

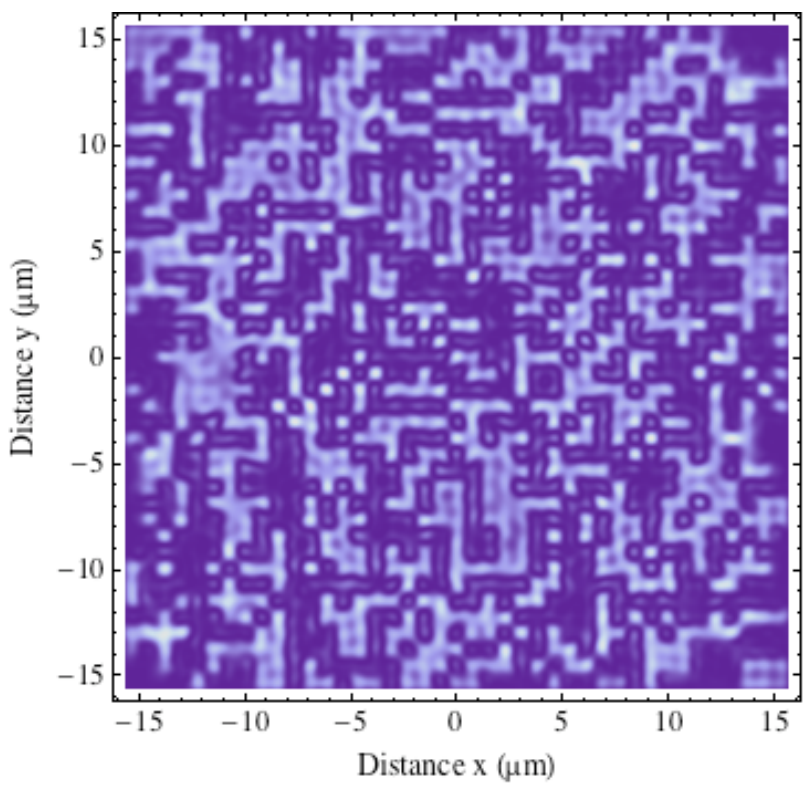

FIG. 4 Density plot of the image retrieved from the diffraction and corresponding to the recorded image shown in Figure 3.

These results demonstrate that the image is retrieved in spite of the small size of the pixel, which is only slightly larger than the optical system resolution, and in spite of the thickness of the material, which is much larger than the depth of focus of this image. One also remarks that all pixels in the retrieved image do not have the same brightness. As discussed above, this brightness depends on the surrounding of the pixel. Typically, pixels on the edges have lower intensities. This non-uniformity of the retrieved pixels is inherent to the Lippmann's architecture. To highlight this feature, we have 
reported, in Figures 5 and 6, 3D plots of the centres of the images shown in Figures 3 and 4. In Figure 6, we notice small bumps at the positions of the "OFF" pixels. They originate from diffraction due to the limited numerical aperture. They are also present on the original image filtered by this aperture, although their height is more limited. They disappear if the pixel size is increased. We also remark that the intensity of the "ON" pixels varies from pixel to pixel. This variation corresponds to the distortion as discussed above.

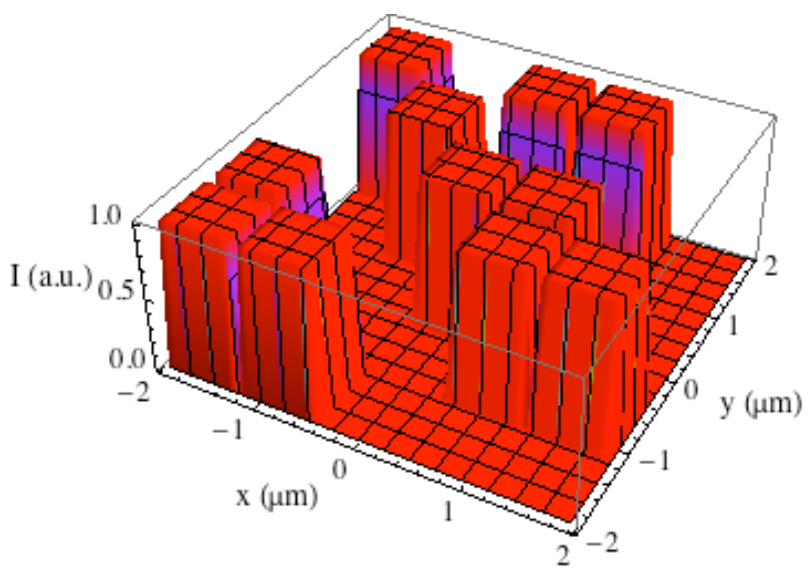

FIG. $53 \mathrm{D}$ plot of the centre of the image shown in Figure 3.

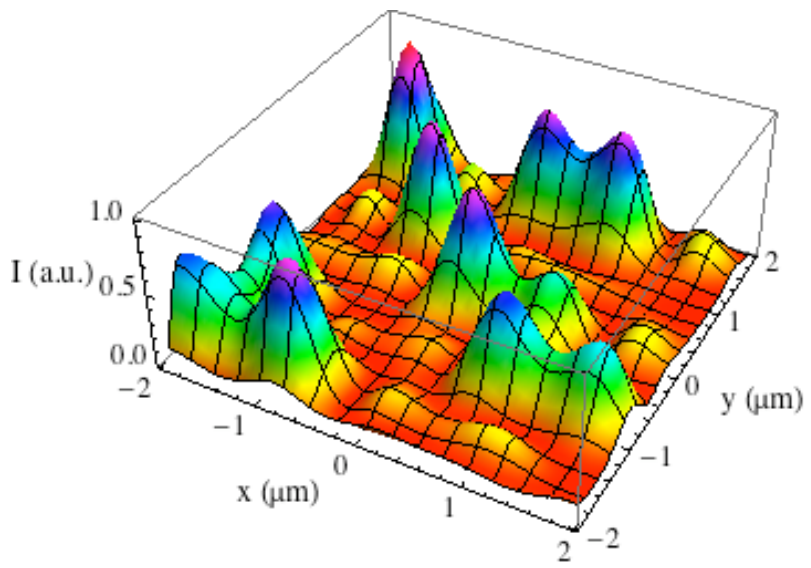

FIG. 63 D plots of the centre of the diffracted image shown in Figure 4.

From these data, we compute the intensity received by each pixel of the detector array (corresponding to each pixel of the SLM, as discussed above). This intensity can either corresponds to a pixel which was set at the " 1 " value, ON pixel, during recording or to the " 0 " value, OFF pixel, during recording. These intensities are denoted by $I_{O N}$ and $I_{O F F}$. The histogram corresponding to these data is plotted in Figure 7.

We then compute the mean values $\mu_{O N / O F F}$ and the standard deviations $\sigma_{O N / O F F}$ of $I_{O N}$ and $I_{O F F}$ in order to calculate the signal to noise ratio of the retrieved data.

$$
S N R=\frac{\mu_{O N}-\mu_{O F F}}{\sqrt{\sigma_{O N}^{2}+\sigma_{O F F}^{2}}}
$$

We found $S N R=4.0$ from these data. If we do not take into account the rows of pixels on the edges, whose intensities are on the average lower, this value increases to $S N R=4.4$. This signal to noise ratio is also increased by increasing the pixel size. For instance, with a pixel side of $0.8 \mu \mathrm{m}$ we find $S N R=$

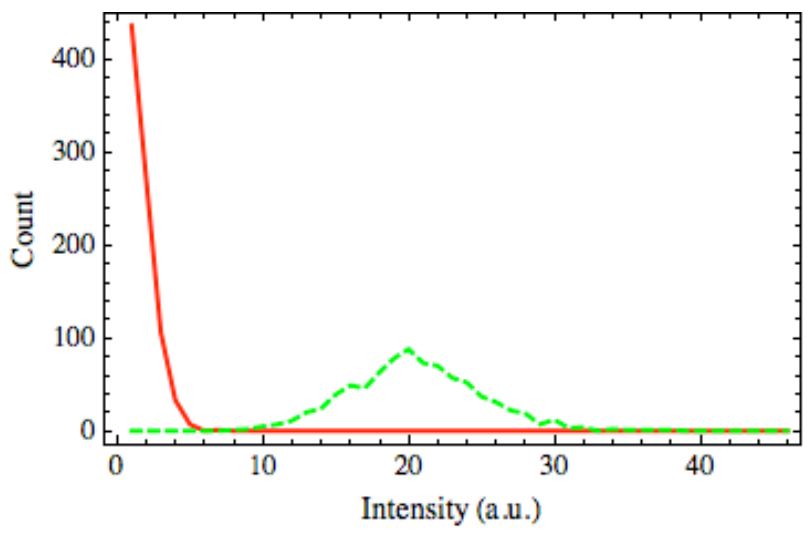

FIG. 7 Histogram of the "OFF" (red continuous line) and "ON" (green dashed line) pixels of the images plotted in Figures 3 and 4.

4.1 with all pixels, and $S N R=4.5$ with the rows on the edges dropped.

A simulation with the same set of parameters, but with a dielectric permittivity proportional to the interference pattern (linear response) gives a lower $S N R, S N R=2.8$, which increases to $S N R=3.1$ by not taking into account the pixels on the edges.

It is important to remind, that, although we call this expression signal to noise ratio, this value is fully deterministic, and depends on the chosen image. Because of this deterministic nature, equalization techniques $[22,23]$ could probably be implemented to greatly improved this signal to noise ratio. The intensity detected on each pixel indeed depends on its surrounding leading to "intersymbol interference". For example, ON pixels, which are on the border of the image, tend to present a lower intensity than pixels in the middle of the image.

It is interesting to note that it is not mandatory to set the sensitive material in contact with the mirror: a gap can be let in between the sensitive layer and the mirror. This gap can be larger than the depth of focus of the image if required. For instance by letting a $10 \mu \mathrm{m}$ gap between the mirror and the $e=25 \mu \mathrm{m}$ thick sensitive layer, we found a $S N R$ equal to $S N R=3.2$, while with a zero gap and a $25 \mu \mathrm{m}$ thick material the $S N R$ equals $S N R=4.0$. Such a gap can greatly ease the implementation of the system:

- the mirror is not necessarily glued to the sensitive layer and can be more easily removed;

- optical elements, such as wave plates, can be inserted in this space allowing an easy implementation of elaborated detection schemes using polarized light such as homodyne detection schemes [16, 18].

The price to pay for allowing such a gap is a stronger requirement on the coherence length of the optical source.

For sake of comparison, all simulated results reported above were conducted with the same set of data (corresponding to Figure 3). We checked that these results do not significantly 
depend on the selected data set. For several data sets selected at random, the average value of the signal to noise ratio is $\langle S N R\rangle \approx 4.2$ with the standard deviation $\sigma_{S N R} \approx 0.1$.

\subsection{Bragg selectivity}

In Lippmann's storage, data are recorded using wavelength multiplexing. It is therefore of first importance to determine the wavelength selectivity to get the minimum required spacing between the two consecutive recording wavelengths. If the images were uniform, and according to Kogelnik's theory for thick gratings [24], the wavelength Bragg selectivity, would be given by:

$$
\Delta \lambda_{\text {Bragg }} \approx \lambda^{2} / 2 n e
$$

with $n$ the refractive index, $\lambda$ the optical wavelength in the vacuum and $e$ the recording layer thickness. $\Delta \lambda_{\text {Bragg }}$ corresponds to the wavelength spacing between the Bragg wavelength (recording wavelength) and the first null of the curve "diffraction efficiency versus wavelength".

We first tested our simulation software with such a plane wave (i.e. uniform and not pixellated beam) for the image amplitude. The readout amplitude is thus equal to this plane wave. We still have $n=1.6, \lambda=500 \mathrm{~nm}$ and $e=30 \mu \mathrm{m}$. The diffracted intensity versus the wavelength is plotted as crosses in Figure 8. The continuous thin line is just for guiding the eyes. This curve reproduces the expected dependence of the diffraction efficiency with the wavelength for a uniform grating. The wavelength Bragg selectivity is conformed to Eq. (6) and equals to $\Delta \lambda_{\text {Bragg }} \approx 2.6 \mathrm{~nm}$.

We then ran the simulation software for a random pixellated image. The corresponding curve is plotted as open squares in Figure 8, the dashed thick line is once again only a guide for the eyes. Although these points do not exactly overlap with the curve for the uniform grating, the similarity is very large. We conclude that even if Lippmann gratings are not uniform through the material thickness, Eq. (6) is a very good approximation for the Bragg selectivity. Therefore the capac-

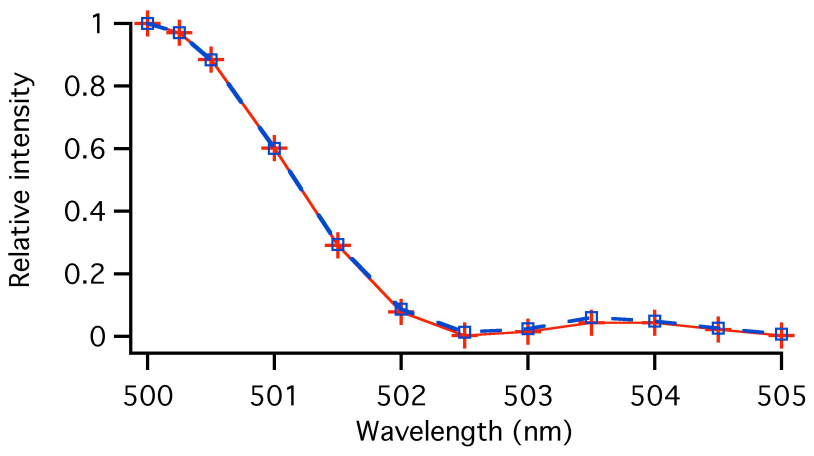

FIC. 8 Comparison of the Bragg selectivity for a plane wave grating (continuous red line and crosses) and for a Lippmann's structure (dashed blue line and open squares).

ity of Lippmann's storage using wavelength multiplexing is the same as for conventional holographic data storage using wavelength multiplexing $[25,26]$.

\section{CONCLUSION}

The main objective of this communication was to demonstrate the possibilities of the Lippmann's approach for optical data storage. We demonstrated that the spatial resolution is just limited by the optical imaging set-up (numerical aperture), that the wavelength selectivity is about the same as for plane waves, and that the signal to noise ratios are reasonable. As these signal to noise ratios result from a deterministic distortion of the images, they can without any doubt be improved using conventional techniques such as image equalizations $[22,23]$ and modulation coding $[27,28]$.

Lippmann's architecture should thus lead to the same capacities as those of conventional holographic approaches. The other inherent advantages of Lippmann's architectures (very stable and compact interferometric set-up, minimization of the required coherence length) make this approach all the more attractive.

Therefore, although Lippmann's photographic process has never attracted a large interest in the field of optical storage, we hope to have contributed in this paper to demonstrate that this technique presents a great richness and that it just awaits to be fully exploited.

\section{ACKNOWLEDGEMENTS}

Kevin Contreras gratefully acknowledges the support of the Programme Alban, the European Union Programme of High Level Scholarships for Latin America (E07D401978PE).

\section{References}

[1] "Holographic Data Storage" in Springer Series in Optical Sciences, H.J. Coufal, D. Psaltis, and G.T. Sincerbox, eds., (Springer-Verlag, 2000).

[2] See for instance the roadmap of the "International Storage Symposium on Optical Memory and Optical Data Storage" available at http://www.isom.jp/.

[3] S.S. Orlov, W. Phillips, E. Bjornson, Y. Takashima, P. Sundaram, L. Hesselink, R. Okas, D. Kwan, and R. Snyder, “High-Transfer-Rate High-Capacity Holographic Disk Data-Storage System" Appl. Optics 43, 4902-4914 (2004).

[4] G. Lippmann, "La photographie des couleurs" C.R. Hebd. Acad. Sci. 112, 274-275 (1891).

[5] G. Lippmann, "Sur la théorie de la photographie des couleurs simples et composées par la méthode interférentielle" J. Phys. (France) 3, 97-107 (1894).

[6] P. Connes, "Silvers salts and standing waves: the history of interference colour photography" J. Opt. 18, 147-166 (1987).

[7] J.M. Fournier, and P.L. Burnett, “Color rendition and archival properties of Lippmann photographs", J. Imaging Sci. Techn. 38, 507-512 (1994).

[8] Y.N. Denisyuk, "The imaging of the optical properties of an object in a wave field of radiation scattered by it" Opt. Spectrosc. 15, 279-284 (1963). 
[9] Y.N. Denisyuk, “Imaging properties of light intensity waves: the development of the initial Lippmann ideas" J. Opt. 22, 275-280 (1991).

[10] K. Tanaka, M. Hara, K. Tokuyama, K. Hirooka, K. Ishioka, A. Fukumoto, and $\mathrm{K}$. Watanabe, "Improved performance in coaxial holographic data recording" Opt. Express 15, 16196-16209 (2007).

[11] S. Orlic, S. Ulm, and H. J. Eichler, " $3 \mathrm{D}$ bit-oriented optical storage in photopolymers" J. Opt. A: Pure Appl Op 3, 72-81 (2001).

[12] C. Denz, G. Pauliat, and G. Roosen, "Volume hologram multiplexing using a deterministic phase encoding method" Opt. Commun. 85 , 171-176 (1991).

[13] H. Fleisher, P. Pengelly, J. Reynolds, R. Schools, and G. Sincerbox, "An optically accessed memory using the Lippmann process for information storage" in Optical and Electro-Optical Information Processing (MIT Press, 1965).

[14] A.S. Hoffman, "Optical information storage in three-dimensional media using the Lippmann Technique" Appl. Optics 1949-1954 (1968).

[15] A. Labeyrie, J.P. Huignard, and B. Loiseaux, "Optical data storage in microfibers" Opt. Lett. 23, 301-303 (1998).

[16] G. Maire, G. Pauliat, and G. Roosen, "Homodyne detection readout for bit-oriented holographic memories" Opt. Lett. 31, 175-177 (2006).

[17] J.-J. Yang, and M.-R. Wang, "White light micrograting multiplexing for high density data storage" Opt. Lett. 31, 1304-1306 (2006).

[18] F. Guattari, G. Maire, K. Contreras, C. Arnaud, G. Pauliat, G. Roosen, S. Jradi, and C. Carré, "Balanced homodyne detection of Bragg microgratings in photopolymer for data storage" Opt. Express 5 , 2234-2243 (2007).
[19] P. Wu, Z. Liu, J.J. Yang, A. Flores, and M.R. Wang, “Wavelengthmultiplexed submicron holograms for disk-compatible data storage" Opt. Express 15, 17798-17804 (2007).

[20] M. Mansuripur, "Distribution of light at and near the focus of high-numerical-aperture objectives" JOSA A 3, 2086-2093 (1986).

[21] P. Várhegyi, P. Koppa, F. Ujhelyi, and E. Lorincz, "System modeling and optimization of Fourier holographic memory" Appl. 0pt. 44, 3024-3031 (2005).

[22] K.M. Chugg, X. Chen, and M.A. Neifeld, "Two-dimensional equalization in coherent and incoherent page-oriented optical memory" J. Opt. Soc. Am. 16, 549-562 (1999).

[23] M. Keskinoz, and B.V.K.V. Kumar, "Discrete magnitude-squared channel modelling, equalization, and detection for volume holographic storage channels" Appl. Optics 43, 1368-1378 (2004).

[24] H. Kogelnik, "Coupled wave theory for thick hologram gratings" ATCTT Tech. J. 48, 2909-2947 (1969).

[25] K. Curtis, C. Gu, and D. Psaltis, "Cross talk in wavelength multiplexed holographic memories" Opt. Lett. 18, 1001-1003 (1993).

[26] G.A. Rakuljic, V. Leyva, and A. Yariv, "Optical data storage using orthogonal wavelength multiplexed volume holograms" Opt. Lett. $17,1471-1473$ (1992).

[27] G.W. Burr, J. Ashley, H. Coufal, R.K. Grygier, J.A. Hoffnagle, C.M. Jefferson, and B. Marcus, "Modulation coding for pixel-matched holographic data storage" Opt. Lett. 22, 639-641 (1997).

[28] G.W. Burr, C.M. Jefferson, H. Coufal, M. Jurich, J.A. Hoffnagle, R. M. Macfarlane, and R. M. Shelby, "Volume holographic data storage at an areal density of 250 gigapixels/in. ${ }^{2 "}$ Opt. Lett. 26, 444-446 (2001). 